\title{
Non-uniform Expansions of Real Numbers
}

\author{
Jörg Neunhäuserer
}

\begin{abstract}
We introduce and study non-uniform expansions of real numbers, given by two non-integer bases.
\end{abstract}

Mathematics Subject Classification. 11K55, 37A45, $28 \mathrm{~A} 78$.

Keywords. Expansions of real numbers, non-inter bases, cardinality of expansions, unique expansions.

\section{Introduction}

Expansions of reals numbers in non-integer bases are studied since the pioneering works of Rényi in the end of the 1950s and Parry in the 1960s, see [11-13]. In these works, especially the greedy algorithm that determines the digits of such expansions and the relationship of these expansions to symbolic dynamics is addressed. In the 1990s, a group of Hungarian mathematics led by Paul Erdős revived this file of research, see [3-5]. Beside other results, they proved that each $x \in(0,1 /(1-q))$ has a continuum of expansions of the form $\sum_{i=1}^{\infty} q^{-n_{i}}$ if $1<q<G$, where $G$ is the golden ratio. In the sequel, Sidorov [14] used ergodic theoretical methods to prove that for all $q \in(1,2)$ almost all $x \in(0,1 /(1-q))$ have such an expansion. Moreover, Glendinning and Sidorov [7] proved that there always exist (at least countably many) reals having a unique expansion if $q>G$. Nowadays, especially dimensional theoretical aspects of expansions of reals numbers in non-integer bases are studied; see, for instance, $[1,9,10]$. In this paper, we introduce non-uniform expansions of real numbers, which may be viewed as expansions with respect to two non-integer bases. As far as we know, such expansions were not studied yet, although they constitute a natural generalisation. The rest of the paper is organized as follows: In the next section, we give two descriptions of nonuniform expansions of real numbers. In the following, we introduce a greedy, a lazy, and intermittent algorithms that give the digits of these expansions. In section four, we prove a theorem on the existence of a continuum of nonuniform expansions of real numbers, which is similar to the results in the uniform case we mentioned above. In the last section, we characterise real 
numbers which have a unique non-uniform expansion and prove a theorem on the cardinality of the set of such numbers.

\section{The Expansions}

Let $\Sigma=\{0,1\}^{\mathbb{N}}$ be the set of sequences of zeros and ones. Equipped with the metric:

$$
d\left(\left(s_{i}\right),\left(t_{i}\right)\right)=\sum_{i=1}^{\infty}\left|s_{i}-t_{i}\right| 2^{-i},
$$

$\Sigma$ is a compact, perfect, and totally disconnected space. For $s=\left(s_{i}\right) \in \Sigma$ and $n \in \mathbb{N}$, let $0_{n}(s)$ be the number of zeros and $1_{n}(s)$ be the number of ones in the sequence $\left(s_{1}, \ldots, s_{n}\right)$.

Fix $\beta_{0}, \beta_{1} \in(1 / 2,1)$ with $\beta_{0} \geq \beta_{1}$ and let $I=I_{\beta_{1}}=\left[0, \beta_{1} /\left(1-\beta_{1}\right)\right]{ }^{1}$ We consider the map $\pi_{\beta_{0}, \beta_{1}}: \Sigma \rightarrow I$ given by:

$$
\pi_{\beta_{0}, \beta_{1}}(s)=\pi_{\beta_{0}, \beta_{1}}\left(\left(s_{i}\right)\right)=\sum_{i=1}^{\infty} s_{i} \beta_{0}^{0_{i}(s)} \beta_{1}^{1_{i}(s)} .
$$

Several times, we will use another description of this map, which we now describe. Let $T_{0}, T_{1}: I \rightarrow I$ be the contractions given by:

$$
T_{0}(x)=\beta_{0} x \quad \text { and } \quad T_{1}(x)=\beta_{1} x+\beta_{1} .
$$

By induction, we have:

$$
T_{s_{1}} \circ \cdots \circ T_{s_{n}}(x)=\beta_{0}^{0_{n}(s)} \beta_{1}^{1_{n}(s)} x+\sum_{i=1}^{n} s_{i} \beta_{0}^{0_{i}(s)} \beta_{1}^{1_{i}(s)} .
$$

Hence, for all $s \in \Sigma$ and all $x \in I$ :

$$
\pi_{\beta_{0}, \beta_{1}}(s)=\pi_{\beta_{0}, \beta_{1}}\left(\left(s_{i}\right)\right)=\lim _{n \rightarrow \infty} T_{s_{1}} \circ \cdots \circ T_{s_{n}}(x) .
$$

Definition 2.1. We call a sequence $s \in \Sigma$ with $\pi_{\beta_{0}, \beta_{1}}(s)=x$ a $\left(\beta_{0}, \beta_{1}\right)$ expansion of $x \in I$.

The following proposition guarantees the existence of $\left(\beta_{0}, \beta_{1}\right)$-expansions.

Proposition 2.1. The map $\pi_{\beta_{0}, \beta_{1}}$ is continuous and surjective.

Proof. If $d\left(\left(s_{i}\right),\left(t_{i}\right)\right)<2^{-u}$, we have $s_{i}=t_{i}$ for $i=1, \ldots, u$, which implies:

$$
\left|\pi_{\beta_{0}, \beta_{1}}\left(s_{i}\right)-\pi_{\beta_{0}, \beta_{1}}\left(t_{i}\right)\right|<\beta_{0}^{u} \beta_{1} /\left(1-\beta_{1}\right) .
$$

Hence, $\pi_{\beta_{0}, \beta_{1}}$ is continuous. Note that:

$$
T_{0}(I) \cup T_{1}(I)=\left[0, \beta_{0} \beta_{1} /\left(1-\beta_{1}\right)\right] \cup\left[\beta_{1}, \beta_{1} /\left(1-\beta_{1}\right)\right]=I,
$$

since $\beta_{0}+\beta_{1} \geq 1$. This implies that:

$$
\bigcup_{\left(s_{i}\right) \in\{0,1\}^{n}} T_{s_{1}} \circ \cdots \circ T_{s_{n}}(I)=I .
$$

\footnotetext{
${ }^{1}$ In the literature, the uniform case $\beta_{0}=\beta_{1}$ has been studied. Usually, the reciprocal of $\beta_{0}$ resp. $\beta_{1}$ is denoted by $\beta$.
} 
for every $n \geq 1$. Hence, for each $x \in I$, there is sequence $s \in \Sigma$, such that:

$$
x \in T_{s_{1}} \circ \cdots \circ T_{s_{n}}(I),
$$

By the compactness of $\Sigma$, the map $\pi_{\beta_{0}, \beta_{1}}$ is surjective

In the next section, we describe an algorithm which determines one $\left(\beta_{0}, \beta_{1}\right)$-expansion of $x \in I$.

\section{The Greedy and the Lazy Algorithm}

Using the notations of last section, we define a map $G: I \rightarrow I$ by:

$$
\begin{aligned}
G(x) & = \begin{cases}T_{0}^{-1}(x), x \notin T_{1}(I) \\
T_{1}^{-1}(x), x \in T_{1}(I)\end{cases} \\
& = \begin{cases}\beta_{0}^{-1} x, & x \in\left[0, \beta_{1}\right) \\
\beta_{1}^{-1} x-1, & x \in\left[\beta_{1}, \beta_{1} /\left(1-\beta_{1}\right)\right],\end{cases}
\end{aligned}
$$

see Fig. 1. For $x \in I$, we define the greedy expansion $g=g(x)=\left(g_{i}\right) \in \Sigma$ with respect to $\left(\beta_{0}, \beta_{1}\right)$ by

$$
g_{i}=\left\lfloor\beta_{1}^{-1} G^{i-1}(x)\right\rfloor,
$$

where $\lfloor a\rfloor$ is the greatest integer not greater than $a$. We have the following.

Proposition 3.1. For all $x \in I$, the greedy expansion $g(x) \in \Sigma$ with respect to $\left(\beta_{0}, \beta_{1}\right)$ is an $\left(\beta_{0}, \beta_{1}\right)$-expansion of $x$; that $i s, \pi_{\beta_{0}, \beta_{1}}(g(x))=x$.

Proof. If $g_{i}=0$, we have $G^{i-1}(x)<\beta_{1}$, which implies $G^{i-1}(x) \notin T_{1}(I)$ and $G^{i-1}(x) \in T_{g_{i}}(I)$. If $g_{i}=1$, we have $G^{i-1}(x) \geq \beta_{1}$, which implies $G^{i-1}(x) \in T_{1}(I)$; hence, again, $G^{i-1}(x) \in T_{g_{i}}(I)$. By the definition of $G$, we conclude:

$$
x \in T_{g_{1}} \circ \cdots \circ T_{g_{i}}(I)
$$

for all $i \in \mathbb{N}$, but this implies $\pi_{\beta_{0}, \beta_{1}}(g(x))=x$.

To define the lazy expansion, let $L: I \rightarrow I$ be given by:

$$
\begin{aligned}
L(x) & = \begin{cases}T_{0}^{-1}(x), x \in T_{0}(I) \\
T_{1}^{-1}(x), x \notin T_{0}(I)\end{cases} \\
& = \begin{cases}\beta_{0}^{-1} x, & x \in\left[0, \beta_{0} \beta_{1} /\left(1-\beta_{1}\right)\right] \\
\beta_{1}^{-1} x-1, & x \in\left(\beta_{0} \beta_{1} /\left(1-\beta_{1}\right), \beta_{1} /\left(1-\beta_{1}\right)\right],\end{cases}
\end{aligned}
$$

see Fig. 1. For $x \in I$, the lazy expansion $l=l(x)=\left(l_{i}\right) \in \Sigma$ with respect to $\left(\beta_{1}, \beta_{2}\right)$ is given by:

$$
l_{i}=\left\lceil\left(1-\beta_{1}\right)\left(\beta_{0} \beta_{1}\right)^{-1} L^{i-1}(x)\right\rceil-1,
$$

where $\lceil a\rceil$ is the smallest integer not smaller than $a$. We have the following.

Proposition 3.2. For all $x \in I$, the lazy expansion $l(x) \in \Sigma$ with respect to $\left(\beta_{1}, \beta_{2}\right)$ is an $\left(\beta_{0}, \beta_{1}\right)$-expansion of $x$; that $i s, \pi_{\beta_{0}, \beta_{1}}(l(x))=x$. 


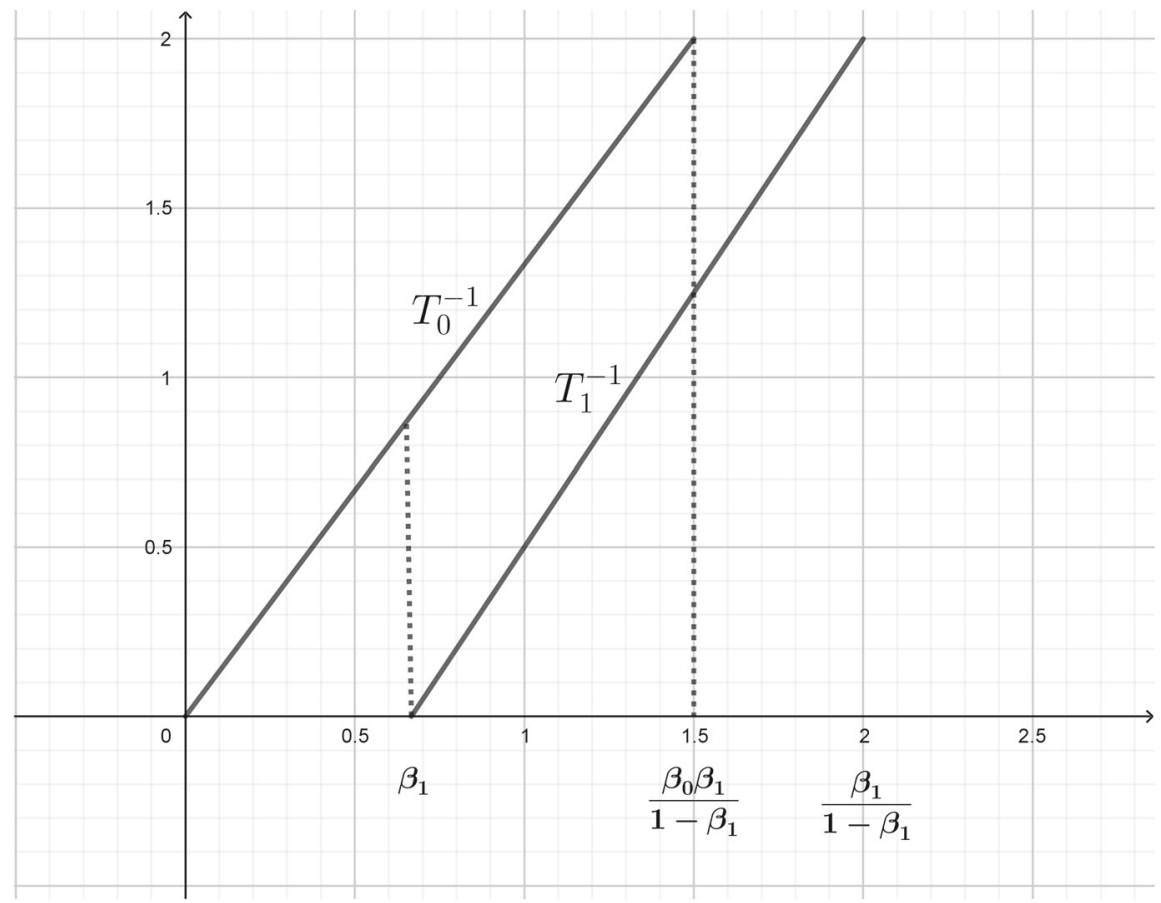

Figure 1. The maps $T_{0}^{-1}$ and $T_{1}^{-1}$ in the case $\beta_{0}=3 / 4, \beta_{1}=$ $2 / 3$

Proof. If $l_{i}=0$, we have $L^{i-1}(x) \leq \beta_{0} \beta_{1} /\left(1-\beta_{1}\right)$, which implies $L^{i-1}(x) \in$ $T_{0}(I)$; hence, $L^{i-1}(x) \in T_{l_{i}}(I)$. If $\bar{l}_{i}=1$, we have $L^{i-1}(x)>\beta_{0} \beta_{1} /\left(1-\beta_{1}\right)$, which implies that $L^{i-1}(x) \notin T_{0}(I)$; hence, again, $L^{i-1}(x) \in T_{l_{i}}(I)$. By the definition of $L$, we conclude:

$$
x \in T_{l_{1}} \circ \cdots \circ T_{l_{i}}(I)
$$

for all $i \in \mathbb{N}$, which implies $\pi_{\beta_{0}, \beta_{1}}(l(x))=x$.

For $\alpha \in\left(\beta_{1}, \beta_{0} \beta_{1} /\left(1-\beta_{1}\right)\right.$, we may also consider intermediate expansions $m(x)=\left(m_{i}\right)$ with respect to $\left(\beta_{1}, \beta_{2}\right)$ given by:

$$
m_{i}=\left\lfloor\alpha^{-1} M_{\alpha}^{i-1}(x)\right\rfloor,
$$

where:

$$
M_{\alpha}(x)=\left\{\begin{array}{ll}
\beta_{0}^{-1} x, & x \in[0, \alpha) \\
\beta_{1}^{-1} x-1, & x \in\left[\alpha, \beta_{1} /\left(1-\beta_{1}\right)\right]
\end{array} .\right.
$$

Again, these are $\left(\beta_{0}, \beta_{1}\right)$-expansions of $x \in I$.

\section{A Continuum of Expansions}

It is natural to ask how many $\left(\beta_{0}, \beta_{1}\right)$-expansions a real number in $I$ has. It turns out that usually there is a continuum of such expansions: 
Theorem 4.1. Let $\beta_{0}, \beta_{1} \in(1 / 2,1)$ with $\beta_{0} \geq \beta_{1}$. We have:

(1) Almost all $x \in I$ have a continuum of $\left(\beta_{0}, \beta_{1}\right)$-expansions.

(2) If $\beta_{1}^{2}+\beta_{0}>1$, all $x \in I \backslash\left\{0, \beta_{1} /\left(1-\beta_{1}\right)\right\}$ have a continuum of $\left(\beta_{0}, \beta_{1}\right)$ expansions.

Proof. We first prove (2). Let $J=\left(0, \beta_{1} /\left(1-\beta_{1}\right)\right)$ and:

$$
\Lambda_{0}=T_{0}(J) \cap T_{1}(J)=\left(\beta_{1}, \beta_{0} \frac{\beta_{1}}{1-\beta_{1}}\right) .
$$

We recursively define $\Lambda_{n+1}=T_{0}\left(\Lambda_{n}\right) \cup \Lambda \cup T_{1}\left(\Lambda_{n}\right)$ and prove by induction:

$$
\Lambda_{n}=\left(\beta_{0}^{n} \beta_{1},\left(\beta_{1}^{n}\left(\beta_{0}-1\right)+1\right) \frac{\beta_{1}}{1-\beta_{1}}\right) \text {. }
$$

We have:

$$
\begin{aligned}
T_{0}\left(\Lambda_{n}\right) & \cup \Lambda_{n} \cup T_{0}\left(\Lambda_{n}\right)=\left(\beta_{0}^{n+1} \beta_{1}, \beta_{0}\left(\beta_{1}^{n}\left(\beta_{0}-1\right)+1\right) \frac{\beta_{1}}{1-\beta_{1}}\right) \\
& \cup\left(\beta_{0}^{n} \beta_{1},\left(\beta_{1}^{n}\left(\beta_{0}-1\right)+1\right) \frac{\beta_{1}}{1-\beta_{1}}\right) \\
& \cup\left(\beta_{0}^{n} \beta_{1}^{2}+\beta_{1},\left(\beta_{1}^{n+1}\left(\beta_{0}-1\right)+1\right) \frac{\beta_{1}}{1-\beta_{1}}\right)=\Lambda_{n+1} .
\end{aligned}
$$

In the last equation, we use $\beta_{1}^{2}+\beta_{0}>1$ and $\beta_{0}^{2}+\beta_{1}>1$, which is true, since $\beta_{0} \geq \beta_{1}$. Note that $\bigcup_{n \geq 0} \Lambda_{n}=J$. Hence, for every $x \in J$, there is a $k \geq 0$ and a sequence $\left(s_{1}, \ldots, s_{k}\right) \in\{0,1\}^{k}$, such that:

$$
x=T_{s_{1}} \circ \cdots \circ T_{s_{k}} \circ T_{0}\left(x_{0}\right) \text { and } x=T_{s_{1}} \circ \cdots \circ T_{s_{k}} \circ T_{1}\left(x_{1}\right),
$$

where $x_{0}, x_{1} \in J$ and $x_{0} \neq x_{1}$. Hence, we obtain two expansions of $x$ that differ in the $k+1$-digit. Applying the result to $x_{0}(x)$ and $x_{1}(x)$, we obtain four expansions of $x$. Here, we use that $x_{0}(x)$ and $x_{1}(x)$ are not at the boundary of $J$. Repeating this procedure $\aleph_{0}$ times, we see that there are $2^{\aleph_{0}}$ expansions of $x$.

Now, we prove (1). Let $G: I \rightarrow I$ be the map associated with the greedy expansion from Sect. 3. $G$ is a piecewise linear expanding interval map and such maps are known to have an ergodic measure, which is equivalent to the Lebesgue measure, see [2] and [8]. By Poincare recurrence theorem for almost all $x \in I$, there is a $k \geq 0$, such that $G^{k}(x) \in \Lambda_{0}$. Hence, for almost all $x \in J$, there is a $k \geq 0$ and a sequence $\left(s_{1}, \ldots, s_{k}\right) \in\{0,1\}^{k}$, such that:

$$
x=T_{s_{1}} \circ \cdots \circ T_{s_{k}} \circ T_{0}\left(x_{0}\right) \text { and } x=T_{s_{1}} \circ \cdots \circ T_{s_{k}} \circ T_{1}\left(x_{1}\right),
$$

where $x_{0}, x_{1} \in J$ and $x_{0} \neq x_{1}$. For almost all $x$, both numbers $x_{1}(x), x_{2}(x)$ have two different $\left(\beta_{0}, \beta_{1}\right)$-expansion, and hence, almost all $x$ have four different expansions. We use here that the intersection of two sets of full measure has full measure. Repeating this procedure $\aleph_{0}$ times, we obtain $2^{\aleph_{0}}$ expansions for almost all $x \in I$, using the fact that a countable intersection of sets of full measure has full measure. 
Obviously, the $\left(\beta_{0}, \beta_{1}\right)$-expansion of 0 and $\beta_{1} /\left(1-\beta_{1}\right)$ is unique. Our theorem leaves the question open if there are numbers $x$ in the interior of $I$ that have a unique $\left(\beta_{0}, \beta_{1}\right)$-expansion. We will address this question in the following section.

\section{Unique Expansions}

We consider the shift map $\sigma:\{0,1\}^{\mathbb{N}} \rightarrow\{0,1\}^{\mathbb{N}}$ given by $\sigma\left(\left(s_{k}\right)\right)=\left(s_{k+1}\right)$. Using this map, we may characterise numbers which have a unique $\left(\beta_{0}, \beta_{1}\right)$ expansion as follows:

Proposition 5.1. The $\left(\beta_{0}, \beta_{1}\right)$-expansion $\left(s_{i}\right)$ of $x$ is unique if and only if:

$$
\pi_{\beta_{0}, \beta_{1}}\left(\sigma^{k}\left(s_{i}\right)\right) \in\left[0, \beta_{1}\right) \cup\left(\beta_{0} \beta_{1} /\left(1-\beta_{1}\right), \beta_{1} /\left(1-\beta_{1}\right)\right]
$$

for all $k \geq 0$.

Proof. $\pi_{\beta_{0}, \beta_{1}}\left(\left(s_{i}\right)\right)=\pi_{\beta_{0}, \beta_{1}}\left(\left(t_{i}\right)\right)$ with $\left(s_{i}\right) \neq\left(t_{i}\right)$ if and only if there exists a smallest $k \geq 0$, such that $s_{k+1} \neq t_{k+1}$ and $\pi_{\beta_{0}, \beta_{1}}\left(\sigma^{k}\left(s_{i}\right)\right)=\pi_{\beta_{0}, \beta_{1}}\left(\sigma^{k}\left(t_{i}\right)\right)$. However, this is equivalent to $\pi_{\beta_{0}, \beta_{1}}\left(\sigma^{k}\left(s_{i}\right)\right) \in T_{0}(I) \cap T_{1}(I)=\left[\beta_{1}, \beta_{0} \beta_{1} /(1-\right.$ $\left.\beta_{1}\right)$. The proposition follows by contraposition.

Using this characterisation of points with unique expansion, we are able to prove:

Theorem 5.1. Let $\beta_{0}, \beta_{1} \in(1 / 2,1)$ and $\beta_{0} \geq \beta_{1}$. (1) If $\beta_{0}\left(1+\beta_{1}\right)<1$, there exist at least countable many $x \in I$, which have a unique $\left(\beta_{0}, \beta_{1}\right)$-expansion. (2) If $\beta_{0}\left(1+2 \beta_{1}-\beta_{0} \beta_{1}\right)<1$, there are uncountable many $x \in I$, which have a unique $\left(\beta_{0}, \beta_{1}\right)$-expansion. Moreover, the set of these $x$ has positive Hausdorff dimension.

Proof. First, we prove (1). Consider the periodic sequence $p=(010101 \ldots)$. Since $\beta_{0}\left(1+\beta_{1}\right)<1$, we have:

$$
\pi_{\beta_{0}, \beta_{1}}(p)=\beta_{0} \beta_{1} /\left(1-\beta_{0} \beta_{1}\right)<\beta_{1} .
$$

Note that $\beta_{0}\left(1+\beta_{1}\right)<1$ implies $\beta_{1}\left(1+\beta_{0}\right)<1$, since $\beta_{0} \geq \beta_{1}$. Hence, we have $\beta_{0}-\beta_{0}^{2} \beta_{1}<1-\beta_{1}$ and thus:

$$
\pi_{\beta_{0}, \beta_{1}}(\sigma(p))=\beta_{1} /\left(1-\beta_{0} \beta_{1}\right)=\beta_{0} \beta_{1} /\left(\beta_{0}-\beta_{0}^{2} \beta_{1}\right)>\beta_{0} \beta_{1} /\left(1-\beta_{1}\right) .
$$

By proposition $x=\pi_{\beta_{0}, \beta_{1}}(p)$ has a unique $\left(\beta_{0}, \beta_{1}\right)$-expansion. Obviously, the same is true for all $x$ of the form $x=\pi_{\beta_{0}, \beta_{1}}((0 \ldots 0101010 \ldots))$, and there exist countable many of such $x$. Now, we prove (2). Let $V=\{01,10\}^{\mathbb{N}}$ and:

$$
U=\bigcup_{k=0}^{\infty} \sigma^{k}(V)=V \cup(\{0\} \times V) \cup(\{1\} \times V),
$$

where $\times$ is the cartesian product. We prove that $\pi_{\beta_{0}, \beta_{1}}(U) \subseteq\left[0, \beta_{1}\right) \cup$ $\left(\beta_{0} \beta_{1} /\left(1-\beta_{1}\right), \beta_{1} /\left(1-\beta_{1}\right)\right]$. The sequence $s \in U$ with $s_{1}=0$ that has the largest projection under $\pi_{\beta_{0}, \beta_{1}}$ obviously is $s=(011010101 \ldots)$. We have:

$$
\pi_{\beta_{0}, \beta_{1}}(s)=\beta_{1} \frac{\beta_{0}+\beta_{0} \beta_{1}-\beta_{0}^{2} \beta_{1}}{1-\beta_{0} \beta_{1}}<\beta_{1}
$$


by our assumption. The sequence $s \in U$ with $s_{1}=1$ that has the smallest projection under $\pi_{\beta_{0}, \beta_{1}}$ obviously is $s=(1001010101 \ldots)$. We have:

$$
\pi_{\beta_{0}, \beta_{1}}(s)=\beta_{1}+\frac{\left(\beta_{0} \beta_{1}\right)^{2}}{1-\beta_{0} \beta_{1}}>\frac{\beta_{0} \beta_{1}}{1-\beta_{1}} .
$$

The inequality here is equivalent to $\beta_{1}\left(1+2 \beta_{0}-\beta_{0} \beta_{1}\right)<1$ which is true, since we assume $\beta_{0} \geq \beta_{1}$. It remains to show that the Hausdorff dimension of $A:=\pi_{\beta_{0}, \beta_{1}}(V)$ is positive. Consider the maps:

$$
F(x)=T_{0} \circ T_{1}(x)=\beta_{0} \beta_{1} x+\beta_{0} \beta_{1}
$$

and

$$
H(x)=T_{1} \circ T_{0}(x)=\beta_{0} \beta_{1} x+\beta_{1},
$$

and let $\Lambda=\left(\pi_{\beta_{0}, \beta_{1}}\left((01)^{\infty}\right), \pi_{\beta_{0}, \beta_{1}}\left((10)^{\infty}\right)\right.$. We have $F(\Lambda) \subseteq \Lambda$ and $H(\Lambda) \subseteq \Lambda$ and $F(\Lambda) \cap H(\Lambda)=\emptyset$ by our assumptions on $\beta_{0}$ and $\beta_{1}$. In the language of fractal geometry, this means that $(F, H)$ induce an iterated function system fulfilling the open set condition, see [6]. The attractor of this iterated function system is $A$, since $A=F(A) \cup H(A)$, and the classical formula for self-similar fractals gives:

$$
\operatorname{dim}_{H} A=\frac{-\log (2)}{\log \left(\beta_{0} \beta_{1}\right)}>0 .
$$

Funding Open Access funding enabled and organized by Projekt DEAL.

Open Access. This article is licensed under a Creative Commons Attribution 4.0 International License, which permits use, sharing, adaptation, distribution and reproduction in any medium or format, as long as you give appropriate credit to the original author(s) and the source, provide a link to the Creative Commons licence, and indicate if changes were made. The images or other third party material in this article are included in the article's Creative Commons licence, unless indicated otherwise in a credit line to the material. If material is not included in the article's Creative Commons licence and your intended use is not permitted by statutory regulation or exceeds the permitted use, you will need to obtain permission directly from the copyright holder. To view a copy of this licence, visit http:// creativecommons.org/licenses/by/4.0/.

Publisher's Note Springer Nature remains neutral with regard to jurisdictional claims in published maps and institutional affiliations.

\section{References}

[1] Barrera, R.A., Baker, S., Kong, D.: Entropy, topological transitivity, and dimensional properties of unique q-expansions. Trans. Am. Math. Soc. 371(5), 3209-3258 (2019)

[2] Denker, M.: Introduction to analysis of dynamical systems. Springer, Berlin (2005) 
[3] Erdös, P., Joo, I., Komornik, V.: Characterization of the unique expansions $\sum_{i=1}^{\infty} q^{-n_{i}}$ and related problems. Bull. Soc. Math. Fr. 118, 377-390 (1990)

[4] Erdös, P., Joó, I.: On the number of expansions $\sum_{i=1}^{\infty} q^{-n_{i}}$. Ann. Univ. Sci. Budapest 35, 129-132 (1992)

[5] Erdös, P., Joó, I., Komornik, V.: On the number of $q$-expansions. Ann. Univ. Sci. Budapest Eötvös Sect. Math. 37, 109-118 (1994)

[6] Falconer, K.: Fractal geometry: mathematical foundations and applications. Wiley, New York (1990)

[7] Glendinning, P., Sidorov, N.: Unique representations of real numbers in noninteger bases. Math. Res. Lett. 8, 535-543 (2001)

[8] Katok, A., Hasselblatt, B.: Introduction to the Modern Theory of Dynamical Systems. Cambridge University Press, Cambridge (1995)

[9] Komornik, V., Kong, D., Li, W.: Hausdorff dimension of univoque sets and devil's staircase. Adv. Math. 305, 165-196 (2017)

[10] Kong, D., Li, W., Lü, F., de Vries, M.: Univoque bases and Hausdorff dimension. Monatsh. Math. 184(3), 443-458 (2017)

[11] Parry, W.: On the $\beta$-expansions of real numbers. Acta Math. Acad. Sci. Hung. 11, 401-416 (1960)

[12] Parry, W.: Representations for real numbers. Acta Math. Acad. Sci. Hung. 15, 95-105 (1964)

[13] Rényi, A.: Representations for real numbers and their ergodic properties. Acta Math. Acad. Sci. Hung. 8, 477-493 (1957)

[14] Sidorov, N.: Almost every number has a continuum of $\beta$-expansions. Amer. Math. Mon. 110, 838-842 (2003)

Jörg Neunhäuserer

Technische Universitat Braunschweig

Braunschweig

Germany

e-mail: joerg.neunhaeuserer@web.de

Received: April 2, 2020.

Revised: July 17, 2020.

Accepted: February 9, 2021. 